\title{
Changes in brain morphology and microstructure in relation to early brain activity in extremely preterm infants
}

\author{
Maria Luisa Tataranno ${ }^{1}$, Nathalie H.P. Claessens ${ }^{1}$, Pim Moeskops ${ }^{1,2}$, Mona C. Toet ${ }^{1}$, Karina J. Kersbergen ${ }^{1}$, \\ Giuseppe Buonocore ${ }^{3}$, Ivana Išgum², Alexander Leemans ${ }^{2}$, Serena Counsell ${ }^{4}$, Floris Groenendaal ${ }^{1}$, Linda S. de Vries ${ }^{1}$ and \\ Manon J.N.L. Benders ${ }^{1}$
}

BACKGROUND AND OBJECTIVE: To investigate the relation of early brain activity with structural (growth of the cortex and cerebellum) and white matter microstructural brain development.

METHODS: A total of 33 preterm neonates (gestational age $26 \pm 1$ weeks) without major brain abnormalities were continuously monitored with electroencephalography during the first $48 \mathrm{~h}$ of life. Rate of spontaneous activity transients per minute (SAT rate) and inter-SAT interval (ISI) in seconds per minute were calculated. Infants underwent brain magnetic resonance imaging $\sim 30$ (mean 30.5; min: 29.3-max: 32.0) and 40 (41.1; 40.0-41.8) weeks of postmenstrual age. Increase in cerebellar volume, cortical gray matter volume, gyrification index, fractional anisotropy (FA) of posterior limb of the internal capsule, and corpus callosum (CC) were measured.

RESULTS: SAT rate was positively associated with cerebellar growth $(P=0.01)$, volumetric growth of the cortex $(P=0.027)$, increase in gyrification $(P=0.043)$, and increase in FA of the CC $(P=0.037)$. ISI was negatively associated with cerebellar growth $(P=0.002)$.

CONCLUSIONS: Increased early brain activity is associated with cerebellar and cortical growth structures with rapid development during preterm life. Higher brain activity is related to FA microstructural changes in the $C C$, a region responsible for interhemispheric connections. This study underlines the importance of brain activity for microstructural brain development.

$\mathbf{T}$ he preterm brain is highly vulnerable in the first postnatal weeks of life, and maintaining structural and functional brain integrity during this critical period is one of the main challenges in neonatal health care. The assessment of early brain activity is an important biomarker of functional brain development in preterm-born neonates $(1,2)$. In early life myelination of the thalamocortical axons is guided by spontaneous electrical signals, and electroencephalography
(EEG) is a useful tool for the assessment of the functional status of these connections. The filtered and time-compressed amplitude-integrated EEG (aEEG) is a bedside tool available for continuous monitoring of brain function in the neonatal unit. Increased brain activity on aEEG is recognized as bursts of high voltage with rapid oscillations, interrupting a period of inactivity $(3,4)$. These high voltage bursts are known as spontaneous activity transients (SATs) and are thought to be crucial for the establishment of brain networks (1). SATs have unique and specific characteristics and can be used as representation of activity in different brain areas and they are necessary for a normal brain. In somatosensory regions, SATs are associated with limb movements in mammalian fetuses and infants (5). In the primary visual cortex, SATs are the expression of spontaneous retinal waves, and are abolished by eye removal (6). In humans, visual SATs abruptly disappear $\sim 35$ weeks of gestation (7). A previous study in our center showed that increased electrical brain activity, or less cortical electrical quiescence, in the first postnatal days correlated with faster volumetric growth of different brain structures up to term equivalent age (TEA) (8). The hypothesized correlation of brain functional and structural maturation creates an early potential interesting predictor of altered brain development.

However, the effect of early brain activity on microstructural brain development has not been established. Fractional anisotropy (FA) is related to fiber coherence, axonal density, and degree of white matter organization. FA of the white matter typically increases with brain maturation and correlates with long-term neurodevelopment $(9,10)$.

White matter, cerebral cortex, and cerebellum are the largest and most vulnerable brain structures in extremely preterm neonates. Furthermore, a developmental relation is suggested between the cerebral cortex and the white matter (11), as well as between the cerebral cortex and the cerebellum (12). The aim of this study was to examine the structural development of the cerebral cortex and cerebellum of the

${ }^{1}$ Department of Neonatology, Wilhelmina Children's Hospital, and Brain Center Rudolf Magnus, University Medical Center Utrecht, Utrecht, The Netherlands; ${ }^{2}$ Image Sciences Institute, University Medical Center Utrecht, Utrecht University, Utrecht, The Netherlands; ${ }^{3}$ Department of Molecular and Developmental Medicine, University of Siena, Siena, Italy; ${ }^{4}$ Centre for the Developing Brain, King's College London, London, UK. Correspondence: Manon J.N.L. Benders (m.benders@umcutrecht.nl)

The first two authors share first authorship.

Received 8 July 2017; accepted 27 November 2017; advance online publication 17 January 2018. doi:10.1038/pr.2017.314 
preterm brain, as well as the microstructural development of white matter in relation to early brain activity.

\section{MATERIALS AND METHODS}

\section{Study Population}

All neonates with a gestational age (GA) at birth below 28 weeks, born between May 2008 and March 2013, and admitted to the Neonatal Intensive Care Unit of the Wilhelmina Children Hospital (Utrecht, The Netherlands) received, according to clinical protocol, aEEG monitoring during the first three days of life and magnetic resonance imaging (MRI) of the brain $\sim 30$ weeks postmenstrual age (PMA), and again around TEA. Infants with congenital malformations, genetic disorders, or metabolic diseases were excluded. Infants with severe brain injury $(n=20)$ were also excluded, as severe brain injury is known to affect the reliability of MRI analysis. Morphine administration is also known to interfere with early brain activity (13). Assessing this effect was beyond the scope of this study, and therefore neonates receiving morphine during aEEG registration were excluded as well $(n=10)$. The total number of inclusions and exclusions can be found in Supplementary Figure S1 online. The medical ethical review committee gave permission for use of the clinical data for research purposes.

\section{Clinical Parameters}

Clinical parameters were obtained by chart review. Birth weight (BW) $z$-scores were computed using the Dutch population reference data (14). Bronchopulmonary dysplasia was considered present in case of oxygen dependency at 36 weeks of GA. Hypoglycemia was defined as a whole-blood glucose level $<2.5 \mathrm{mmol} / \mathrm{l}$. Hypotension was defined as mean arterial blood pressure $(\mathrm{mm} \mathrm{Hg})$ of less than the GA (in weeks) at that time. Arterial blood pressure was measured using an indwelling arterial catheter. Severe brain injury (an exclusion criterion) was defined as the presence of an intraventricular hemorrhage grade III or IV (according to Papile et al. (15)), posthemorrhagic ventricular dilatation (ventricular index $>97$ th percentile) (16), periventricular leukomalacia grade II or III (17), or a large cerebellar hemorrhage $(>3 \mathrm{~mm})$. White matter injury was assessed by two neonatologists (L.S.d.V. and M.J.N.L.B.) using the Kidokoro scoring system (18).

\section{aEEG Acquisition}

Bedside aEEG at a sampling rate of $256 \mathrm{~Hz}$ was started as soon as possible after birth and continued for at least $48 \mathrm{~h}$. Subcutaneous needle electrodes were used with a central reference electrode measuring the impedance. The P3-P4 cross-sectional signal was chosen, and is known to be predictive of neurodevelopment in preterm infants (19). Owing to technical reasons (impedance, inhomogeneity of data, and differences in filters) only infants monitored with BrainZ monitors (BRM2/BRM3, BrainZ; Natus, Seattle, WA) were enrolled (Supplementary Figure S1).

\section{EEG Postregistration Analysis}

In-house-developed software (SignalBase v7.8, University Medical Center, Utrecht, The Netherlands) was used to process the raw EEG data. In all patients three epochs of $1 \mathrm{~h}$ were manually selected at the aEEG at three specific postnatal time points: $20-24 \mathrm{~h}$ (T1), 32-36 h (T2), and $44-48 \mathrm{~h}$ (T3). The aEEG records were visually assessed to identify artifacts, caretaking events, and periods with high impedance, to select the best hour per epoch.

Quantitative analysis with the same software program obtained the number of SATs per minute (SAT rate) (rounded to whole number) and the inter-SAT interval (ISI, i.e. time between SAT) in seconds per minute, both derived from the raw EEG. SAT and ISI, the socalled event-based EEG measures, are explained in Figure 1a, b. The quantification of SAT and ISI was done using a nonlinear energy operator (http://iopscience.iop.org/0967-3334/31/11/N02). In the present cohort, seven patients were registered with BRM 2 and 26 patients with BRM 3 monitors. These devices had a filter setting of $2 \mathrm{~Hz}$ and $0.5 \mathrm{~Hz}$, respectively, which implied a lower sensitivity of BRM 2 monitors for low frequencies. Ten patients monitored with BRM3 were randomly selected and resampled with a $2 \mathrm{~Hz}$ filter. The results were compared with the $0.5 \mathrm{~Hz}$ filter and no significant differences were found in EEG parameters.

\section{MRI Acquisition}

MRI was performed on a 3T MR system (Achieva; Philips Medical Systems, Best, The Netherlands). At 30 weeks, PMA, an MRIcompatible incubator, was used to reduce patients discomfort and maintain temperature (Dräger MR Incubator, Lübeck, Germany and Nomag IC 3.0, Lammers Medical Technology GmbH, Lübeck, Germany, with a dedicated neonatal head coil). At TEA, infants were positioned within a vacuum pillow in a SENSE head coil. The protocol included T2-weighted imaging in the coronal plane (turbo

a

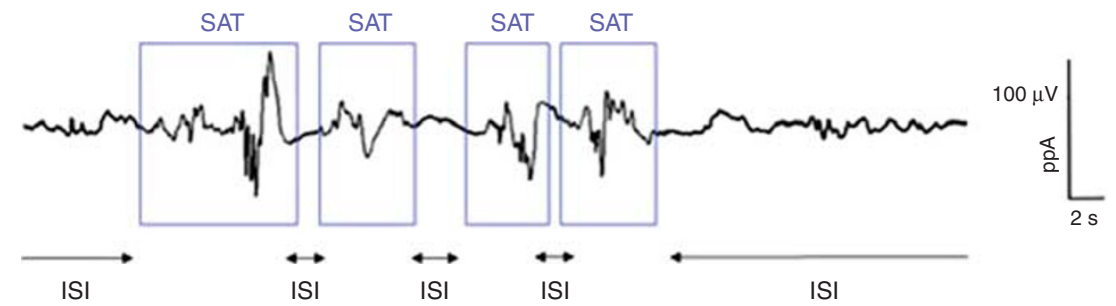

b
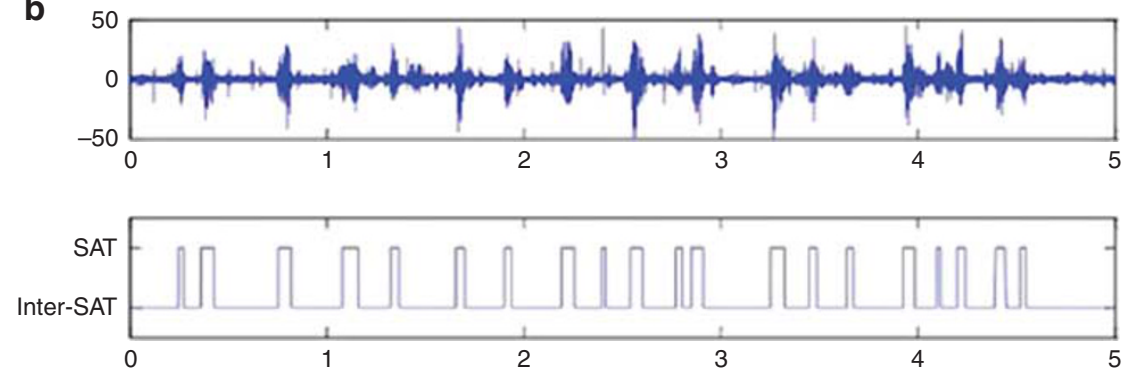

Figure 1. Event-based electroencephalography (EEG) measures. (a) An example of a $30 \mathrm{~s}$ EEG record of one infant showing several spontaneous activity transients (SATs) with the inter-SAT intervals in between (ISI). (b) An epoch of 5 min EEG with below detected SAT and ISI. 


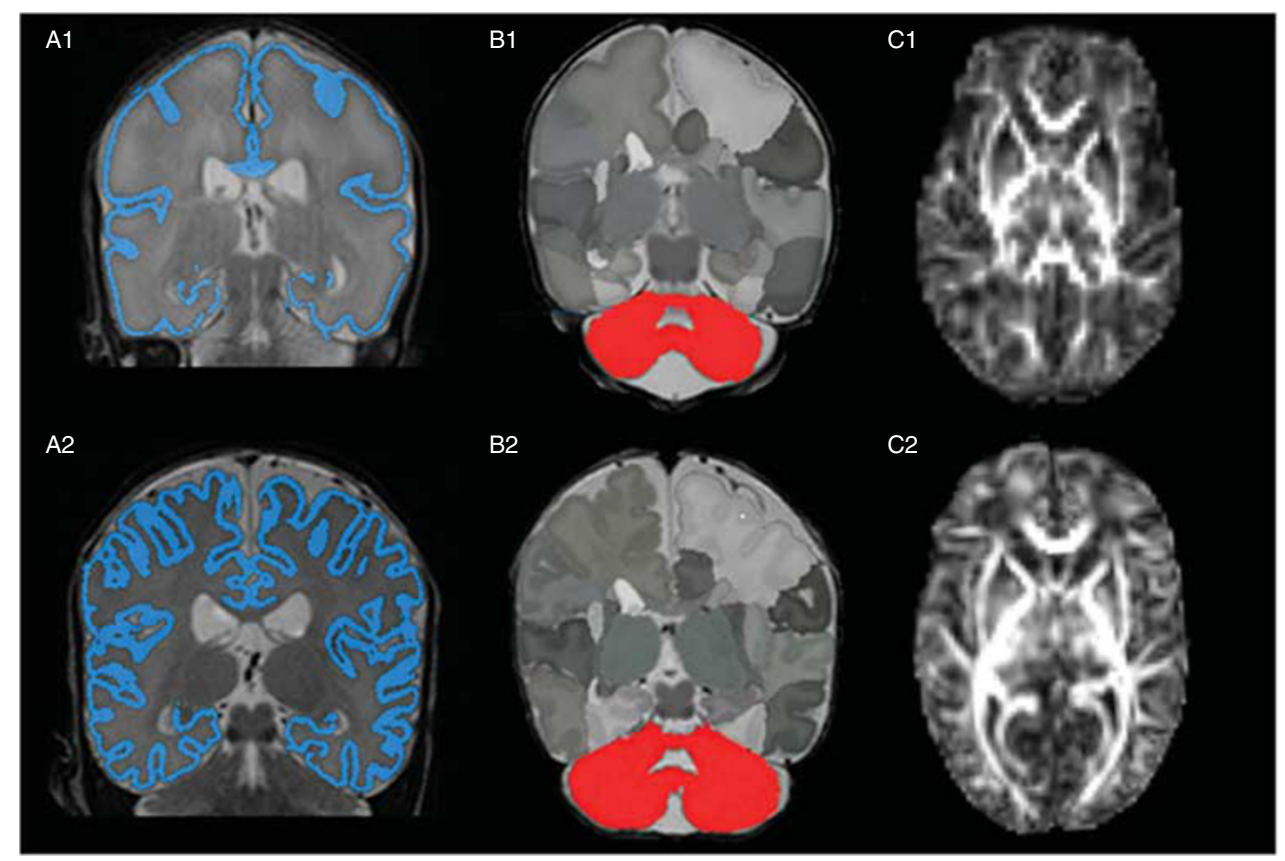

Figure 2. Examples of image analysis results. The top row shows the 30 weeks examples, the bottom row shows the 40 weeks examples. (a 1 and a2) Examples of the method of Moeskops et al. (16) used for the cortical quantification analysis. (b1 and b2) Examples of the method of Makropoulos et al. (14) used to obtain the cerebellar volume. (c1 and c2) Examples of the diffusion tensor imaging (DTI) registration (http://www. exploredti.com/) used to obtain the fractional anisotropy (FA) of the posterior limb of the internal capsule and corpus callosum.

spin echo, at 30 weeks: repetition time 10,085 ms; echo time $120 \mathrm{~ms}$; slice thickness $2 \mathrm{~mm}$, in-plane spatial resolution $0.35 \times 0.35 \mathrm{~mm}^{2}$; at TEA: repetition time 4,847-6,293 ms; echo time 120-150 ms; slice thickness $1.2 \mathrm{~mm}$, in-plane spatial resolution $0.35 \times 0.35 \mathrm{~mm}^{2}$, full brain coverage) and diffusion tensor imaging (DTI) in the axial plane (single-shot spin-echo, repetition time $5,685 \mathrm{~ms}$, echo time $70 \mathrm{~ms}$, field of view $180 \times 146 \mathrm{~mm}^{2}, 32$ diffusion-weighted images with $B$ value of $800 \mathrm{~s} / \mathrm{mm}^{2}$, and one non-diffusion-weighted image; matrix size $128 \times 102 \mathrm{~mm}^{2}$, slice thickness $2 \mathrm{~mm}$, full brain coverage). During the whole examination a neonatologist was present. Oxygen saturation, respiratory, and heart rate of the infants were monitored. If necessary, the infants were sedated using oral chloral hydrate $30 \mathrm{mg} / \mathrm{kg}$ at 30 weeks PMA and $50-60 \mathrm{mg} / \mathrm{kg}$ at TEA. All infants received double-layer hearing protection using Minimuffs (Natus Medical Incorporated, San Carlos, CA) and Earmuffs (EM's 4 Kids, Brisbane, QLD, Australia).

\section{MRI Analysis}

T2-weighted scans were used to segment the cerebellum with an automated segmentation method (20), which allowed computation of cerebellar volume. This method has shown reliable results among infants scanned between 28 and 44 weeks PMA. Results of all segmentations were visually inspected and small corrections were performed if deemed necessary. Examples are provided in Figure $\mathbf{2 b}$.

To enable analysis of the cortex, another automated segmentation method was used for the segmentation of the cortical gray matter using T2-weighted images (21). This method allowed computation of cortical gray matter volume $\left(\mathrm{cm}^{3}\right)$ - characterizing cortical growthand gyrification index-characterizing cortical maturation (22). Gyrification index was measured as the ratio between inner cortical surface and a smooth convex hull around the white matter (i.e., a simulated cortex around the brain without any folds). Example results of cortical gray matter segmentations are shown in Figure 2a.

Quality assessment and analysis of DTI images were performed with the diffusion MRI toolbox ExploreDTI (http://www.exploredti. $\mathrm{com} /$ ). Data were corrected for eddy current-induced geometric distortions and subject motion as described previously (23). In this procedure, the required reorientation of the B-matrix was performed and the tensor model was fitted to the data with the REKINDLE approach $(23,24)$. The FA values of the posterior limb of the internal capsule (PLIC) and corpus callosum (CC) were computed by registering the images to a neonatal atlas (25) using both affine and elastic transformation (Figure 2c).

\section{Statistical Analysis}

Statistical analysis was performed using IBM SPSS v21.0 (Chicago, IL). The time effect of EEG variables over the three epochs (T1-T2T3) was checked through a linear regression model, and in the absence of time dependency the three epochs were averaged to a single value of SAT rate and ISI. All structural brain volumes at 30 weeks and at TEA were corrected for total brain volume. Growth of the structural brain volumes and increase of gyrification index and FA parameters were measured as growth/increase per 10 weeks. For these calculations, the absolute difference between the measurement at TEA and at 30 weeks of GA was taken, divided by the period (in weeks) between the two scans and multiplied by 10 with the following formula: ((vol 40-vol 30)/weeks between scans) $\times 10$, as already described by Kersbergen et al. (26). All data were corrected for PMA at the time of the MRI. First, a univariable regression analysis was performed to determine which factors had the largest effect on MRI parameters (results shown in Supplementary Table S1. Owing to the small sample size, only the most influential variables were included in the multivariable linear regression analysis. A univariable linear regression analysis was used to determine the effect of clinical confounders on EEG variables (results shown in Supplementary Table S2. The multivariable regression model included GA, BW $z$-score, and white matter injury score and was built to evaluate the associations between quantitative measures of early brain activity and increase of the MRI measures. A separate multivariable model was built per EEG parameter and MRI parameter. A $P$ value below 0.05 was considered statistically significant. 
Table 1. Baseline characteristics of the study population

\begin{tabular}{|c|c|}
\hline Baseline characteristics & $N=33$ \\
\hline Gestational age (weeks), mean (SD) & $26.0(1.0)$ \\
\hline Birth weight, mean (g) (SD) & $916(157)$ \\
\hline SGA (<p10), $n(\%)$ & $2(6)$ \\
\hline Male/female, $n(\%)$ & $12 / 21(36 / 64)$ \\
\hline Apgar score 1 min, median (IQR) & $6(3-7)$ \\
\hline Apgar score 5 min, median (IQR) & $8(7-9)$ \\
\hline $\mathrm{pH}$ arterial/venous cord blood, mean (SD) & $7.27(0.08)$ \\
\hline \multicolumn{2}{|l|}{ Hypoglycemia (<2.5 mmol/l) } \\
\hline Once, $n(\%)$ & $4(12.1)$ \\
\hline Repeated episodes, $n$ (\%) & $1(3)$ \\
\hline \multicolumn{2}{|l|}{ Hypotension $(M A B P<G A)$} \\
\hline Treatment: fluids, $n$ (\%) & $4(12.1)$ \\
\hline Inotropes, $n$ (\%) & $12(36.4)$ \\
\hline GMH-IVH grade I-II, $n$ (\%) & $3(9)$ \\
\hline \multicolumn{2}{|l|}{ Patent ductus arteriosus } \\
\hline Conservatively treated, $n$ (\%) & $16(49)$ \\
\hline Surgically treated, $n(\%)$ & $1(3)$ \\
\hline Necrotizing enterocolitis, $n$ (\%) & $1(3)$ \\
\hline Bronchopulmonary dysplasia, $n$ (\%) & $5(15)$ \\
\hline Culture-proven sepsis, $n$ (\%) & $14(42)$ \\
\hline Punctate cerebellar hemorrhage at TEA, $n(\%)$ & $3(9)$ \\
\hline Mild or moderate WMI, $n$ (\%) & $23(77)$ \\
\hline Weight at 30 weeks scan, mean (g) (SD) & $1,183(190)$ \\
\hline $\mathrm{HC}$ at 30 weeks scan, mean $(\mathrm{cm})(\mathrm{SD})$ & $27(2)$ \\
\hline Weight at 40 weeks scan, mean (g) (SD) & $3,421(388)$ \\
\hline $\mathrm{HC}$ at 40 weeks scan, mean $(\mathrm{cm})(\mathrm{SD})$ & $35(1)$ \\
\hline
\end{tabular}

GA, getational age; GMH, germinal matrix hemorrhage; $H C$, head circumference; IQR, interquartile range; IVH, intraventricular hemorrhage; MAPB, mean arterial blood pressure; SGA, small for gestational age; TEA, term equivalent age; WMI, white matter injury..

\section{RESULTS}

\section{Patients}

In total, 33 infants were eligible for the study, all with good quality aEEG and serial MRI. Clinical characteristics are presented in Table 1. Early MRI was performed at a mean PMA of 30.5 (range 29.3-32.0) weeks and TEA MRI at a mean PMA of 41.1 (range 40.0-41.8) weeks. Growth of the structural and microstructural brain measurements between 30 and 40 weeks is shown in Figure 3. None of the enrolled patients were presented with clinical or laboratory signs of perinatal asphyxia. None of the infants were mechanically ventilated during EEG monitoring. One infant received phenobarbital at the dose of $10 \mathrm{mg} / \mathrm{kg}$ on day 2 because of suspicion of electrical discharges. However, no severe brain injury was observed in this patient. None of the other included infants received any other medication influencing brain activity such as benzodiazepines and barbiturates.

In infants with clinical signs of hypotension needing inotropes, the mean dopamine dose was $5 \mu \mathrm{g} / \mathrm{kg} / \mathrm{min}, \mathrm{SD}$ 2.3 , range $5-12 \mu \mathrm{g} / \mathrm{kg}$ per $\mathrm{min}$. The univariable linear regression analysis on the effect of clinical confounders on EEG variables showed that BW $z$-score was significantly and negatively associated with ISI. (Supplementary Table S2).

\section{Early Brain Activity and Structural Brain Growth}

A significant positive association between SAT rate and cerebellar growth was found with conversely a significant negative association between ISI and cerebellar growth (Table 2 and Figure 4). Other clinical variables did not show an effect to cerebellar growth in the multivariable analyses. SAT rate also was positively correlated with growth of cortical gray matter and increase in gyrification index, where ISI did not show any relation.

A clear example of the clinical relevance of the present study is that an increase of one SAT/min in the first $48 \mathrm{~h}$ after birth will bring an increase of $\sim 0.2 \mathrm{~cm}^{3}$ to the cerebellar volume and of $\sim 5 \mathrm{~cm}^{3}$ of cortical gray matter volume. An increase of one second in ISI will result in a decrease of $\sim 0.14 \mathrm{~cm}^{3}$ in cerebellar volume.

\section{Early Brain Activity and Microstructural Brain Growth}

The multivariable analysis showed a positive association between SAT rate and increase in FA of the CC (Table 3). Thus, an increase in one SAT per minute will result in an increased FA value of the CC of $\sim 0.03$.

No association between ISI and the CC was seen. Also, no associations were found between EEG measurements (SAT and ISI) and increase in FA of the PLIC.

\section{DISCUSSION}

This study shows increased early brain activity (expressed by higher SAT rate and lower ISI) to be associated with increased growth of the cerebral cortex and cerebellum and also with changes in corpus callosal FA, in a relatively healthy cohort of extremely preterm infants. These results underline the importance of early life neuromonitoring in extremely preterm neonates in order to identify those at risk of altered brain development.

This study confirms the importance of SATs for cortical gray matter growth, as presented in a previous paper (8) and adds the importance of early spontaneous activity to cortical maturation (i.e., gyrification). SATs are the most notable feature of the preterm EEG, characterized by nested activity at multiple frequencies, including also very-low-frequency activity $(0.1-0.5 \mathrm{~Hz})$ that is filtered by conventional EEG (1). In healthy human infants, SATs disappear $\sim 35$ weeks of PNA (27). The association between early brain activity (i.e., SAT rate and ISI) and morphological maturation of the cortex between 30 and 40 weeks is thought to be a result of the complex interplay between structural and functional processes in this period of brain network development (28). EEG 


\section{Articles | Tataranno et al.}
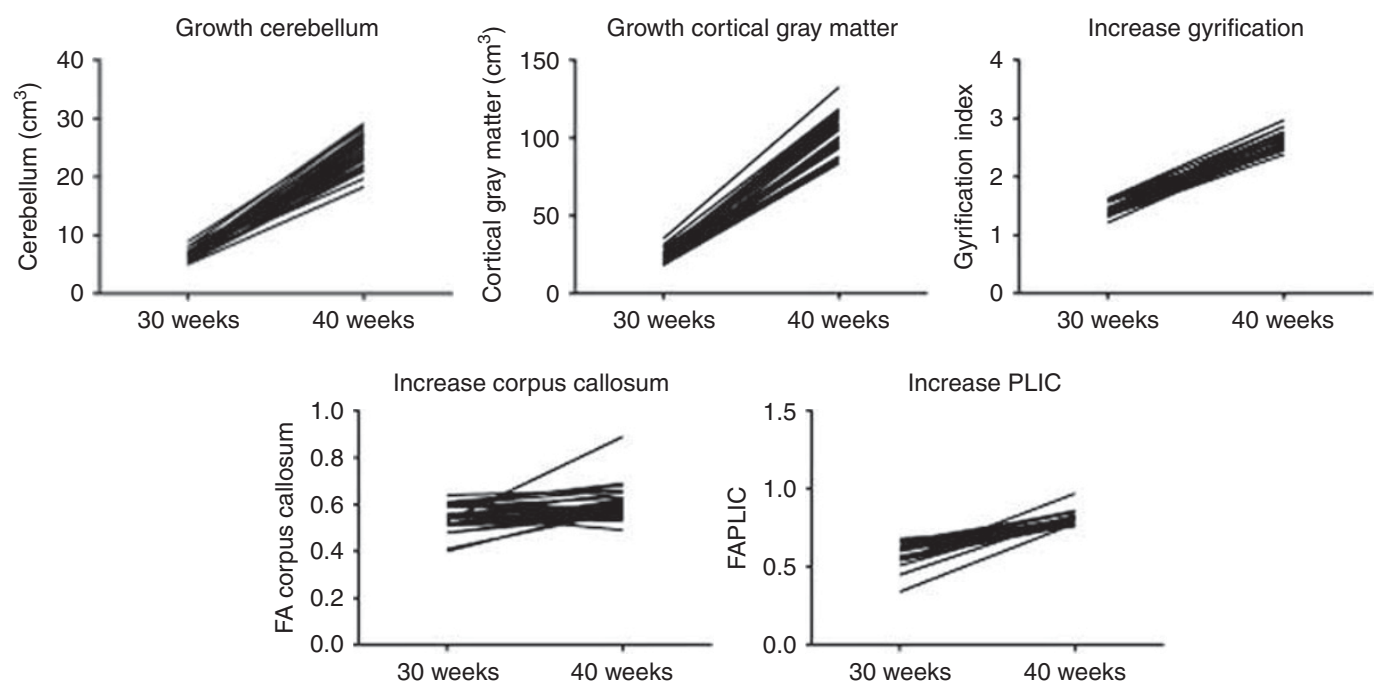

Figure 3. Structural and microstructural brain growth. Increase between 30 and 40 weeks for all 33 individuals are shown for cerebellar volume, cortical gray matter (cGM) volume, gyrification index (GI), corpus callosum (CC) fractional anisotropy (FA), and posterior limb of the internal capsule (PLIC) FA. For each variable mean (SD) and range (;) is provided: cerebellum 30 weeks $\left(\mathrm{cm}^{3}\right)$ : $6.48(0.87)(5.00 ; 8.90), 40$ weeks: 24.56 (2.63) (18.28; 29.16). cGM 30 weeks $\left(\mathrm{cm}^{3}\right)$ : $22.84(3.91)(17.78 ; 35.18), 40$ weeks: $104.57(11.82)(83.65 ; 132.69)$. Gl 30 weeks: $1.40(0.09)(1.21 ; 1.62), 40$ weeks: 2.66 (0.13) $(2.38 ; 2.97)$. FA CC 30 weeks: $0.53(0.06)(0.40 ; 0.64)$, 40 weeks: $0.59(0.08)(0.36 ; 0.89)$. FA PLIC 30 weeks: $0.57(0.07)(0.34 ; 0.68), 40$ weeks: 0.80 (0.04) $(0.69 ; 0.97)$. All the values were corrected age at scan.

Table 2. EEG parameters and structural brain development

\begin{tabular}{|c|c|c|c|c|c|c|}
\hline & \multicolumn{2}{|l|}{ Cerebellum } & \multicolumn{4}{|c|}{ Cortical gray matter } \\
\hline & Volumetric growth, $B(\mathrm{Cl})$ & $P$ value & Volumetric growth, $B(\mathrm{Cl})$ & $P$ value & Gyrification, $B(\mathrm{Cl})$ & $P$ value \\
\hline SAT & $0.234(0.060 ; 0.408)$ & 0.010 & $4.706(0.566 ; 8.847)$ & 0.027 & $0.050(0.002 ; 0.099)$ & 0.043 \\
\hline BW z-score & $-0.008(-0.255 ; 0.239)$ & 0.945 & $0.702(-4.598 ; 6.002)$ & 0.787 & $-0.079(-0.140 ;-0.018)$ & 0.014 \\
\hline WMI score & $0.040(-0.093 ; 0.173)$ & 0.540 & $-1.134(-4.498 ; 2.231)$ & 0.493 & $-0.032(-0.070 ; 0.006)$ & 0.095 \\
\hline GA & $0.022(-0.152 ; 0.195)$ & 0.801 & $1.356(-2.836 ; 5.548)$ & 0.666 & $0.022(-0.029 ; 0.073)$ & 0.374 \\
\hline ISI & $-0.149(-0.237 ;-0.062)$ & 0.002 & $-1.735(-4.049 ; 0.578)$ & 0.135 & $-0.019(-0.047 ; 0.009)$ & 0.178 \\
\hline BW z-score & $-0.100(-0.310 ; 0.109)$ & 0.334 & $0.011(-6.886 ; 6.907)$ & 0.997 & $-0.066(-0.132 ;-0.001)$ & 0.048 \\
\hline WMI score & $-0.020(-0.150 ; 0.110)$ & 0.753 & $-1.134(-4.498 ; 2.231)$ & 0.493 & $-0.022(-0.060 ; 0.016)$ & 0.238 \\
\hline GA & $0.020(-0.162 ; 0.202)$ & 0.827 & $0.626(-4.184 ; 5.435)$ & 0.790 & $0.021(-0.033 ; 0.074)$ & 0.426 \\
\hline
\end{tabular}

BW, birth weight; Cl, confidence interval; EEG, electroencephalography; GA, gestational age; ISI, inter-SAT interval; SAT, spontaneous activity transient; WMI, white matter injury. Rows show the EEG parameters (SAT and ISI) and the other clinical parameters included in the multivariable analyses. Separate multivariable analyses were performed for ISI and SAT. Columns show the growth of cerebellar volume, cortical gray matter volume, and gyrification index. $B$ value with $\mathrm{Cl}$ and $P$ value are shown Volumetric growth is calculated per 10 weeks. Significant results are in bold.

findings are the result of cortical network activity, changing with maturation of the brain during the last part of gestation (29). From 24 weeks onward afferent thalamocortical axons grow from the thalamus into the subplate where accumulation and waiting take place before migration to the cortex. Migration from the subplate into the cortical plate induces synaptogenesis of the thalamocortical axons, which corresponds with the appearance of SATs at EEG (30,31). It is known that SATs are first focal and concentrated in the sensory cortex, and become more widespread later during development (1). Onset of synchronicity between hemispheres can be seen $\sim 30$ weeks of gestation, whereas more precise and consistent synchrony appears only from $\sim 35$ weeks of conceptional age, and it is not completely dependent on callosal connections (32). This study shows increased SAT rate to be associated with cortical gray matter maturation, which is supported by other studies finding more complex EEG patterns with increased gyrification (33). A recent study comparing subjects with no or minimal brain injury to those with moderate/severe brain injury showed that the latter have lower burst frequency and longer IBI and includes data from both rodent and human preterm subjects. In the rodent model of the same study, diminished brain activity was associated with a delayed neuronal morphological development demonstrating that spontaneous brain activity (SATs) is fundamental for normal maturation and 

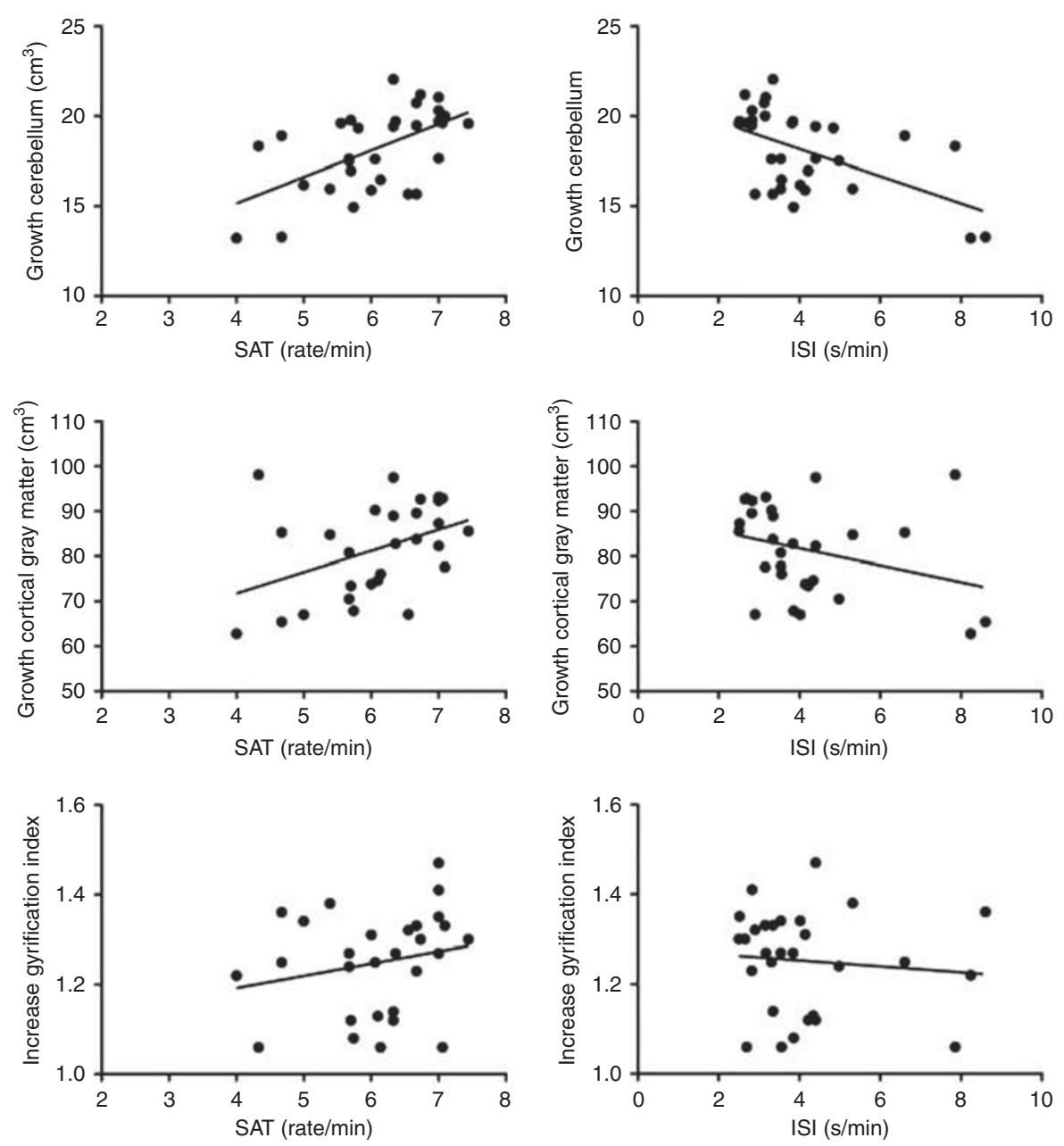

Figure 4. Electroencephalography (EEG) and structural brain growth. Associations of EEG parameters (spontaneous activity transient (SAT) and interSAT interval (ISI)) with cerebellar volume, cortical gray matter volume, and gyrification index are shown. Coefficients and confidence intervals corrected for confounding factors are shown in Table 2; therefore, $R$ and $P$ values are not included in this figure. EEG variables mean (SD) and range (;) are provided as follows: SAT (rate/min): $6.0(0.8)(4.0 ; 7.4)$, ISI (s/min): $4.1(1.6)(2.5 ; 8.6)$.

Table 3. EEG parameters and microstructural brain development

\begin{tabular}{|c|c|c|c|c|}
\hline & \multicolumn{2}{|c|}{ Corpus callosum } & \multicolumn{2}{|c|}{ PLIC } \\
\hline & Increase $\mathrm{FA}, B(\mathrm{Cl})$ & $P$ value & Increase $\mathrm{FA}, B(\mathrm{Cl})$ & $P$ value \\
\hline SAT & $0.027(0.020 ; 0.053)$ & 0.037 & $-0.013(-0.067 ; 0.042)$ & 0.608 \\
\hline WMI score & $-0.012(-0.052 ; 0.027)$ & 0.501 & $-0.105(-0.184 ;-0.027)$ & 0.013 \\
\hline GA & $0.021(-0.010 ; 0.052)$ & 0.168 & $0.053(-0.012 ; 0.117)$ & 0.098 \\
\hline BW z-score & $0.006(-0.052 ; 0.065)$ & 0.811 & $-0.054(-0.120 ; 0.012)$ & 0.098 \\
\hline WMI score & $-0.009(-0.051 ; 0.033)$ & 0.639 & $-0.105(-0.184 ;-0.027)$ & 0.013 \\
\hline GA & $0.030(-0.001 ; 0.060)$ & 0.053 & $0.053(-0.012 ; 0.117)$ & 0.098 \\
\hline
\end{tabular}

BW, birth weight; Cl, confidence interval; EEG, electroencephalography; FA, fractional anisotropy; GA, gestational age; ISI, inter-SAT interval; PLIC, posterior limb of the internal capsule; SAT, spontaneous activity transient; WMI, white matter injury.

Row show the EEG parameters (SAT and ISI) and the other clinical parameters included in the multivariable analyses. Separate multivariable analyses were performed for ISI and SAT. Columns show increase of fractional anisotropy FA in the corpus callosal and PLIC. B value with CI and $P$ value are shown. Microstructural brain development was calculated as growth per 10 weeks. Significant results are in bold. 


\section{Articles | Tataranno et al.}

development of cortical circuits and neurons. These data suggest that early brain injury impairs activity-dependent maturation of neuronal circuits during a highly sensitive period of development (34). We were able to show that increased cerebellar growth is associated with early brain activity, whereas in contrast, low SAT rate and prolonged ISI are associated with poor cerebellar growth. An approximately three times increase in cerebellar volume from 28 to 40 weeks of gestation has been documented, which makes the cerebellum, more than the cortical gray matter, the brain structure showing the largest relative growth in this period (26). This suggests the cerebellar and cortical expansion to be most vulnerable to disturbances (in brain activity) in this early period of life of preterm infants. Several studies have suggested a developmental relation between the cerebellum and cortical gray matter in infants born preterm, where the cortex and cerebellum appear to be connected in a closed circuit with bidirectional feedback, supported by recent studies describing reduced contralateral cortical gray matter growth in case of prematurity-related cerebellar injury (35-37). Both cerebellar and cortical alterations are associated with neurodevelopmental sequelae in the domains of cognition and motor development (35-37). This study, suggesting developmental dependency upon early brain activity of the cerebellum and the cortex, puts forward that cortical alterations might already find their origin in the first period of life in extremely preterm neonates. It cannot be excluded that the importance of brain activity and subsequent brain development is a surrogate of the infant's well-being, such as optimal cardiovascular and nutrition conditions, therefore optimal brain growth.

In this study, we showed prolonged ISI in early life to be associated with decreased cerebellar growth between 30 and 40 weeks. Prolonged ISI (and thus decreased cortical activity) has been associated with acidosis and adverse long-term neurodevelopmental outcome at 2 years of corrected age in preterm infants $(19,38)$. Furthermore, some studies demonstrated that the survival of developing neurons is strongly dependent on the growing spontaneous activity in the early neuronal networks $(39,40)$. Thus, deprivation of neurons of this activity, reflected by prolonged ISI, may be linked to cell apoptosis and reduced dendritic pruning $(41,42)$. Based on our study, we can hypothesize that prolonged ISI in early life has more effect on cerebellar growth than on cortical gray matter development (43).

To the best of our knowledge, we have shown for the first time that increased early brain activity was associated with microstructural changes in the CC, as determined by faster increase in FA. This confirms the hypothesis of a link between early brain function and white matter microstructural maturation. White matter FA increases with brain maturation as a result of increasing myelination, fiber coherence, and axonal density. White matter organization is known to be correlated with long-term neurodevelopmental outcome in this vulnerable group of neonates $(44,45)$. Being the largest white matter fiber bundle, the CC is responsible for the majority of the corticocortical, interhemispheric connections (46). Reduced FA of the CC might reflect altered myelination as well as axonal damage, resulting in decreased interhemispheric processing. The PLIC is one of the WM structures with the largest increase in FA over the period of 30-40 weeks of PMA (47). White matter organization of the PLIC, predictive of motor development, and vulnerable to injury in preterm infants (44) did not show an association with early brain activity in this study. This could be explained by the fact that neonatal morbidity has a stronger effect on the process of maturation of the PLIC, than the effect found by early brain activity.

This study examined a relatively healthy cohort of preterm infants by excluding those who died, were unstable for MRI, received morphine, or had severe brain injury.

The main problem of these neonates is extreme prematurity, and many factors can influence later brain development. This study aimed to take into account as less severe preterm problems as possible by excluding infants with severe brain injury. In this group of vulnerable infants an association between early cortical network activity and brain microstructural development is suggested.

The first limitation of this study is the small population, leading to limited statistical strength. The analysis methods used require high-quality EEG and MRI data, resulting in a limited number of infants eligible for inclusion. However, selection bias does not seem to play a role, as baseline characteristics between included and excluded patients (based on image quality) were comparable. The small sample size results in a limited number of included relevant covariables in the analysis. Thus, the analysis could not be corrected for all confounders on aEEG and could not optimally be controlled for all known variables associated with brain growth. Furthermore, the small sample size yields the possibility to underscore the effect of other confounders.

\section{CONCLUSIONS}

This study demonstrates the association of early brain activity to structural and microstructural brain growth in a cohort of relatively healthy extremely preterm infants. Higher SAT rate and decreased ISI are signs of increased early cortical network activity and appear to be associated with increased growth of cerebellum and cortical gray matter, as also cortical maturation. Cerebellum and cortex undergo the most rapid growth in the last part of gestation and are connected in a closed neuronal system. A change in FA of the CC was also shown to be associated with higher spontaneous brain activity in early preterm life. This study underlines the importance of early brain activity for improved microstructural brain development in extremely preterm infants and the need for neuromonitoring in these vulnerable newborns.

\section{SUPPLEMENTARY MATERIAL}

Supplementary material is linked to the online version of the paper at http://www.nature.com/pr

\section{ACKNOWLEDGMENTS}

This work includes infants participating in the Neobrain study (LSHMCT-2006-036534). The first draft of the manuscript has been written by M.L. T. and N.H.P.C. Part of this study was presented as an abstract at the 5th 


\section{Brain activity and brain development $\quad$ Articles}

Congress of the European Academy of Paediatric Societies (EAPS) 17-21 October 2014, Barcelona (Spain), PS47.

\section{STATEMENT OF FINANCIAL SUPPORT}

The research of A.L. is supported by VIDI Grant 639.072.411 from the Netherlands Organization for Scientific Research (NWO).

Disclosure: The authors declare no conflict of interest.

\section{REFERENCES}

1. Vanhatalo S, Kaila K. Development of neonatal EEG activity: from phenomenology to physiology. Semin Fetal Neonatal Med 2006;11:471-8.

2. Griesmaier E, Enot DP, Bachmann M, et al. Systematic characterization of amplitude-integrated EEG signals for monitoring the preterm brain. Pediatr Res 2013;73:226-35.

3. Toet MC, van Rooij LG, de Vries LS. The use of amplitude integrated electroencephalography for assessing neonatal neurologic injury. Clin Perinatol 2008;35:665-78 v.

4. Hellstrom-Westas L, Rosen I. Continuous brain-function monitoring: state of the art in clinical practice. Semin Fetal Neonatal Med 2006;11: $503-11$.

5. Blumberg MS, Marques HG, Iida F. Twitching in sensorimotor development from sleeping rats to robots. Curr Biol 2013;23:R532-7.

6. Colonnese MT, Khazipov R. "Slow activity transients"in infant rat visual cortex: a spreading synchronous oscillation patterned by retinal waves. J Neurosci 2010;30:4325-37.

7. Colonnese Mt, Kaminska A, Minlebaev M, et al. A conserved switch in sensory processing prepares developing neocortex for vision. Neuron 2010;67:480-98.

8. Benders MJ, Palmu K, Menache C, et al. Early brain activity relates to subsequent brain growth in premature infants. Cereb Cortex 2015;25: 3014-24.

9. Pandit AS, Ball G, Edwards AD, Counsell SJ. Diffusion magnetic resonance imaging in preterm brain injury. Neuroradiology 2013;55 (Suppl 2): 65-95

10. Counsell SJ, Edwards AD, Chew AT, et al. Specific relations between neurodevelopmental abilities and white matter microstructure in children born preterm. Brain 2008;131 (Part 12): 3201-8.

11. Volpe JJ. Brain injury in premature infants: a complex amalgam of destructive and developmental disturbances. Lancet Neurol 2009;8: $110-24$.

12. Limperopoulos C, Chilingaryan G, Sullivan N, Guizard N, Robertson RL, du Plessis AJ. Injury to the premature cerebellum: outcome is related to remote cortical development. Cereb Cortex 2014;24:728-36.

13. Norman E, Wikstrom S, Rosen I, Fellman V, Hellstrom-Westas L. Premedication for intubation with morphine causes prolonged depression of electrocortical background activity in preterm infants. Pediatr Res 2013;73:87-94.

14. Visser GH, Eilers PH, Elferink-Stinkens PM, Merkus HM, Wit JM. New Dutch reference curves for birthweight by gestational age. Early Hum Dev 2009;85:737-44.

15. Papile LA, Burstein J, Burstein R, Koffler H. Incidence and evolution of subependimal and intraventricular hemorrhage: a study of infants with birth weight less than $1500 \mathrm{gm}$. J Pediatr 1978;92:529-34.

16. Brouwer AJ, van Stam C, Uniken Venema M, Koopman C, Groenendaal F, de Vries LS. Cognitive and neurological outcome at the age of 5-8 years of preterm infants with post-hemorrhagic ventricular dilatation requiring neurosurgical intervention. Neonatology 2012;101:210-6.

17. de Vries LS, Eken P, Dubowitz LM. The spectrum of leukomalacia using cranial ultrasound. Behav Brain Res 1992;49:1-6.

18. Kidokoro H, Neil JJ, Inder TE. New MR imaging assessment tool to define brain abnormalities in very preterm infants at term. Am J Neuroradiol 2013;34:2208-14.

19. Wikstrom S, Pupp IH, Rosen I, et al. Early single-channel aEEG/EEG predicts outcome in very preterm infants. Acta Paediatr 2012;101: $719-26$.
20. Makropoulos A, Gousias IS, Ledig C, et al. Automatic whole brain MRI segmentation of the developing neonatal brain. IEEE Trans Med Imag 2014;33:1818-31.

21. Moeskops P, Benders MJ, Chit SM, et al. Automatic segmentation of MR brain images of preterm infants using supervised classification. Neuroimage 2015;118:628-41.

22. Moeskops P, Benders MJ, Kersbergen KJ, et al. Development of cortical morphology evaluated with longitudinal MR brain images of preterm infants. PLoS ONE 2015;10:e131552.

23. Leemans A, Jones DK. The B-matrix must be rotated when correcting for subject motion in DTI data. Magn Reson Med 2009;61:1336-49.

24. Tax CM, Otte WM, Viergever MA, Dijkhuizen RM, Leemans A. REKINDLE: robust extraction of kurtosis INDices with linear estimation. Magn Reson Med 2015;73:794-808.

25. Oishi K, Mori S, Dnohue PK, et al. Multi-contrast human neonatal brain atlas: application to normal neonate development analysis. Neuroimage 2011;56:8-20.

26. Kersbergen KJ, Makropoulos A, Aljabar P, et al. Longitudinal regional brain development and clinical risk factors in extremely preterm infants. J Pediatr 2016;178:e6.

27. Vanhatalo S, Palva JM, Andersson S, Rivera C, Voipio J, Kaila K. Slow endogenous activity transients and developmental expression of $\mathrm{K}^{+}-\mathrm{Cl}-$ cotransporter 2 in the immature human cortex. Eur J Neurosci 2005;22: 2799-804.

28. Smyser CD, Inder TE, Shimony JS, et al. Longitudinal analysis of neural network development in preterm infants. Cereb Cortex 2010;20:2852-62.

29. Natalucci G, Leuchter RH, Bucher HU, et al. Functional brain maturation assessed during early life correlates with anatomical brain maturation at term-equivalent age in preterm infants. Pediatr Res 2013;74:68-74

30. Innocenti GM, Price DJ. Exuberance in the development of cortical networks. Nat Rev Neurosci 2005;6:955-65.

31. Kostovic I, Judas M. The development of the subplate and thalamocortical connections in the human foetal brain. Acta Paediatr 2010;99:1119-27.

32. Scher MElectroencephalography of the newborn: normal and abnormal featuresIn:Niedermeyer E, Lopez da Silva Feds. Electroencephalography: basic principles, clinical applications, and related fields. Boston, Massachussetts: Lippincott-Williams and Wilkins, 2004:937-90.

33. Biagioni E, Frisone MF, Laroche S, et al. Maturation of cerebral electrical activity and development of cortical folding in young very preterm infants. Clin Neurophysiol 2007;118:53-9.

34. Ranasinge S, Or G, Wang EY, et al. Reduced cortical activity impairs development and plasticity after neonatal hypoxia ischemia. J Neurosci 2015;35:11946-59.

35. Limperopoulos C, Bassan H, Gauvreau K, et al. Does cerebellar injury in premature infants contribute to the high prevalence of long-term cognitive, learning, and behavioral disability in survivors? Pediatrics 2007;120:584-93.

36. Messerschmidt A, Brugger PC, Boltshauser E, et al. Disruption of cerebellar development: potential complication of extreme prematurity. Am J Neuroradiol 2005;26:1659-67.

37. Dubois J, Benders M, Borradori-Tolsa C, et al. Primary cortical folding in the human newborn: an early marker of later functional development. Brain 2008;131 (Part 8): 2028-41.

38. Eaton DG, Wertheim D, Oozeer R, Dubowitz LM, Dubowitz V. Reversible changes in cerebral activity associated with acidosis in preterm neonates. Acta Paediatr 1994;83:486-92.

39. Hanganu-Opatz IL. Between molecules and experience: role of early patterns of coordinated activity for the development of cortical maps and sensory abilities. Brain Res Rev 2010;64:160-76.

40. Colonnese M, Khazipov R. Spontaneous activity in developing sensory circuits: implications for resting state fMRI. Neuroimage 2012;62:2212-21.

41. Nimmervoll B, White R, Yang JW, et al. LPS-induced microglial secretion of TNFalpha increases activity-dependent neuronal apoptosis in the neonatal cerebral cortex. Cereb Cortex 2013;23:1742-55. 


\section{Articles | Tataranno et al.}

42. Tolner EA, Sheikh A, Yukin AY, Kaila K, Kanold PO. Subplate neurons promote spindle bursts and thalamocortical patterning in the neonatal rat somatosensory cortex. J Neurosci 2012;32:692-702.

43. Petanjek Z, Judas M, Simic G, et al. Extraordinary neoteny of synaptic spines in the human prefrontal cortex. Proc Natl Acad Sci USA 2011;108:13281-6.

44. van Kooij BJ, de Vries LS, Ball G, et al. Neonatal tract-based spatial statistics findings and outcome in preterm infants. Am J Neuroradio 2012;33:188-94.
45. Ment LR, Hirtz D, Huppi PS. Imaging biomarkers of outcome in the developing preterm brain. Lancet Neurol 2009;8:1042-55.

46. Schmahmann JD, Pandya DN, Wang R, et al. Association fibre pathways of the brain: parallel observations from diffusion spectrum imaging and autoradiography. Brain 2007;130 (Part 3): 630-53.

47. Kersbergen KJ, Leemans A, Groenendaal F, et al. Microstructural brain development between 30 and 40 weeks corrected age in a longitudinal cohort of extremely preterm infants. Neuroimage 2014;103: 214-24. 